\title{
Lab automation: tales along the road to automation
}

\section{The benefits of laboratory automation do not come without considerable effort and patience in setting up the systems. Nathan Blow talks with two groups at different stages on the road to automation.}

Robotic liquid dispensers, plate handlers, automated plate readers and scanners are just a few of the instruments designed to make life in the lab simpler for scientists. And although these technologies open new research doors for many labs, there is also a learning curve to lab automation. "The thing that really struck me is that it may be a labor-saving devicebut it requires a lot of work to set up," says Jennifer Rohn of University College London when discussing her recent experience in setting up a liquid-dispensing robot.

Still, more and more labs, both large and small, are starting to use automated solutions to enhance their workflows. To really understand the benefits and the drawbacks involved in lab automation, it is helpful to obtain the insights of both expert users and beginners.

\section{A high-throughput approach to life sciences}

When it comes to developing and using high-throughput automated approaches to research, the Genomics Institute of the Novartis Research Foundation (GNF) is an impressive example. GNF was started in 1999 as a technology research center. "There was a realization that high-throughput technologies could have a big impact on scientific discovery and ultimately the translation of basic science to medicine," says Peter Schultz, founder and institute director of GNF. But at the time GNF was founded, Schultz says, people had different pieces of these highthroughput technologies-screening systems, mRNA and protein expression and analysis systems - but no one had yet assimilated the tools and used them synergistically. "GNF was created with the vision of bringing all of these technologies that enable various aspects of life sciences together."

"When I was at Berkeley, Pete Schultz talked about starting a center that would do high-throughput arrays, chemical genomics,

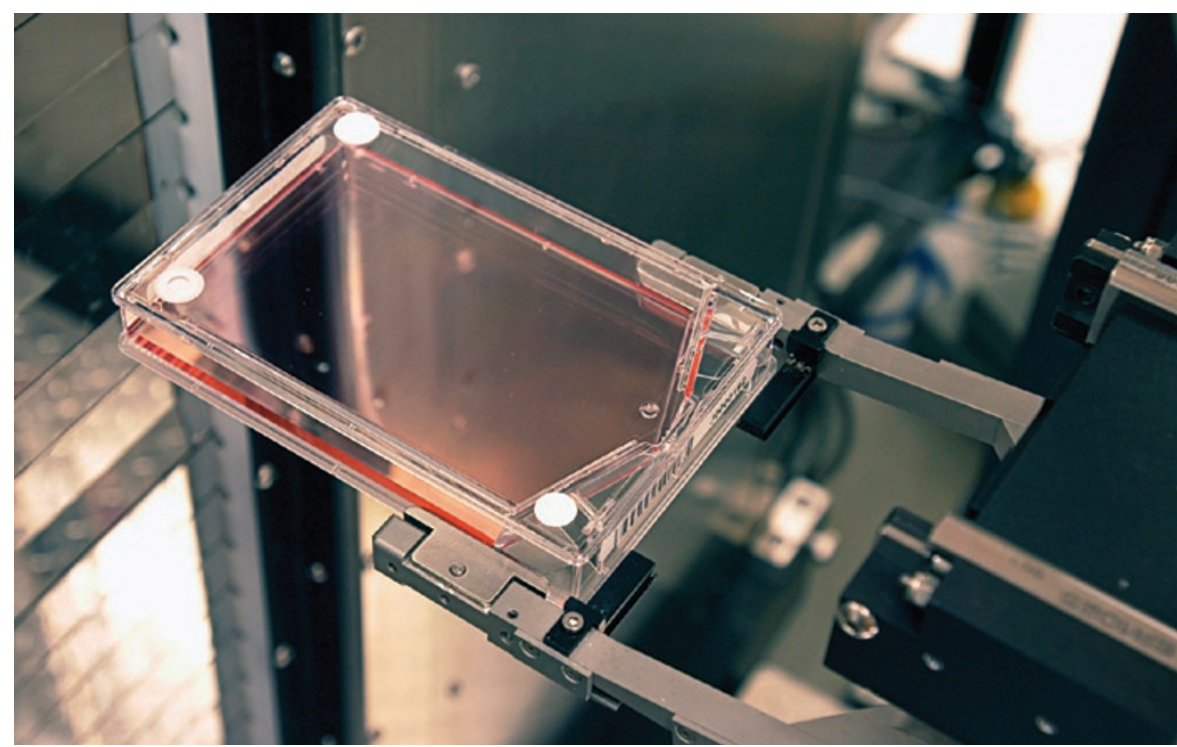

Flask culture modified for robotic use. (Courtesy of GNF.)

functional genomics and structural genomics," says Mike Cooke, director of immunology at GNF. "I took one look at him and said, "If you could do two or even three of those, it would be great'." Cooke says that today automated technologies have been developed in each of these areas at GNF, giving the institute a solid, high-throughput toolbox to explore biology at a level of detail few others are capable of.

Anthony Orth is a group leader at GNF who performs full-genome small interfering RNA screens using automation developed at GNF and a sixfold coverage human small interfering RNA library. Orth's group worked with GNF engineers to develop a robot specifically designed for genome screening that would not only set up all the transfections, but also move plates between liquid handlers, incubators and plate readers for the final assay output. "We have run close to 200 genomic screens of 60,000100,000 wells on this system," says Orth.
The final readout can be done using either an integrated Perkin Elmer ViewLux plate reader for fluorescence-based assays or an Opera 384-well confocal system that can image cells directly. Having two different plate readers available for use with the robot can be a benefit. "Sometimes it is crucial to look at the cellular phenotype, since this can give you very different information," notes Orth.

Whereas Orth and his group use robotics for full genome-wide screens, Scott Lesley heads another GNF group using highthroughput robotic methods for structural biology. "We set out to use all the genomic information being generated and do highthroughput structural biology," says Lesley. And to this end, Lesley's group has created a pipeline for cloning genes into a wide array of expression vectors. After this step the robots take over and perform expression and purification from bacterial cells, baculoviruses and even mammalian cells. 


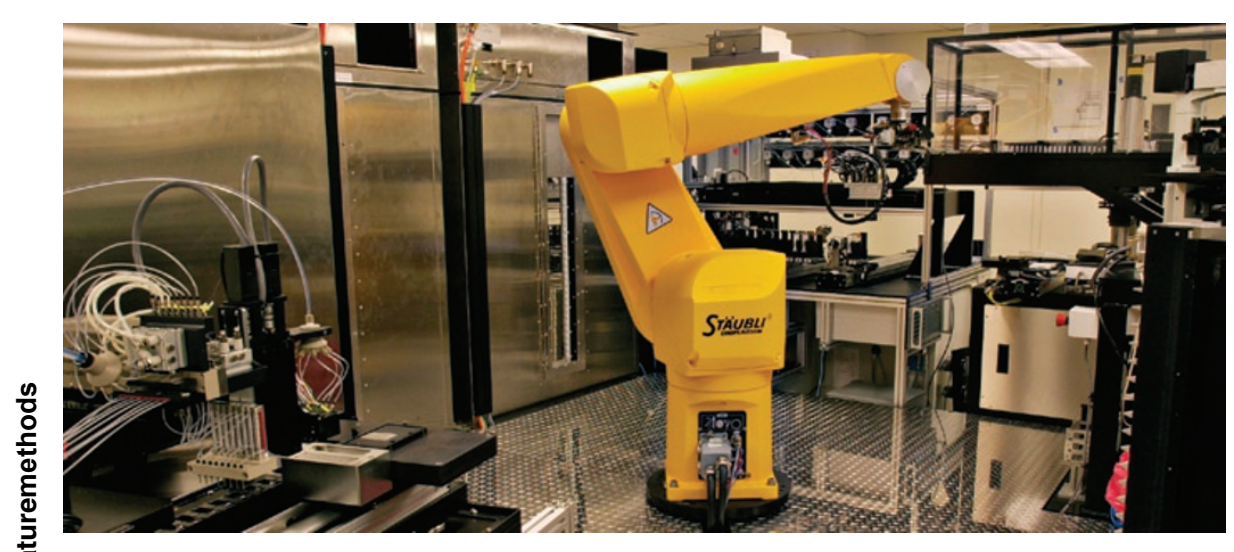

GNF develops systems for automated cell profiling and compound screening. (Courtesy of GNF.)

Bacterial protein expression is done using a parallel fermentation system composed of 96 arrayed 100-ml culture tubes that allow high-density cell growth with yields of $2-4 \mathrm{~g}$ of cell pellet for each culture, with minimal variation. The protein purification is done on GNF's automated protein purification system, which comprises a 96-tube centrifuge, sonication probes and liquid-handling and affinity-purification capabilities. "Purifications typically yield around $10 \mathrm{mg}$ of purified protein per tube and take about 6 hours for 96," said Lesley.

But for the newest protein expression system-designed for mammalian and insect cells-GNF's engineers had not only to design a new robotic platform, but also to develop an entirely new culture flask to work with the robotics. The new flask is thin, with a rubber septum at the top for inoculation; allows for growth of adherent cells or cells in suspension; and has a pocket at the bottom to collect cell pellets after centrifugation.

The GNF group integrated a Tecan robotic platform to transfect mammalian cells or virally infect insect cells (using a baculovirus-based system). Primary protein purification is automated and integrated into the robotics platform and uses cell lysis, high-speed centrifugation and affinity purification.

The initial results from this system are impressive-it has capacity for 900 individual protein expressions and purifications per week, although the actual throughput depends on how long the incubations take. But Lesley notes that it is quite reasonable to obtain 300 proteins per day. From there the proteins are used in several different automated analytical assays, including aggregation testing, identification of posttranslational modifications, ligand-bind- ing assays, activity testing and structure determination. Lesley's group can also make proteins for drug-screening applications - at a very large scale. "We have made grams of proteins for some screens," he says.

\section{Expert users bring development in house}

In 2002, GNF moved into the area of drug discovery. "The goal was to rapidly capture the knowledge from human genomics and use it for drug discovery," says Avi Spier, director of business development at GNF. And it was the advancements in robotics made during the early years at GNF that set the stage for the institute's automated approach to drug discovery.

"In 1999, there was no 1,536-well robotic capability that worked well, so we brought it in house to develop," says Bob
Downs, executive director of engineering, informatics and information technology for GNF. Downs, a former head engineer for the carmaker Saturn, along with Daniel Sipes, director of advanced automation applications, and colleagues decided to apply the quality control and durability standards of the robots used in car manufacturing to biological screens. This meant fabricating almost all robotic parts in-house and having a stock center on hand with a large inventory of materials parts. "An engineer can put something together, but if you do not have good quality control, everything from the liquid dispensing to the results of the screens will be different," warns Downs. Using this philosophy, Downs and Sipes have developed robotic capabilities at GNF for compound screening optimized for the 1,536well plate format.

The cornerstone of the drug discovery effort at GNF is the ACP, or automated compound profiler. These massive, roomsize robotic systems consist of multiple large Staubli robotic arms that move cell culture flasks plates between numerous modified climate-controlled incubators, several large, enclosed carousels holding GNF's two-million-compound library arrayed out in 1,536-well plates, liquid dispensers and washers specially designed in-house by GNF engineers for cell-based assays in 1,536-well plates, and different plate readers including the ViewLux and Lumilux and the Flipr Tetra from Molecular Devices for fluorescence

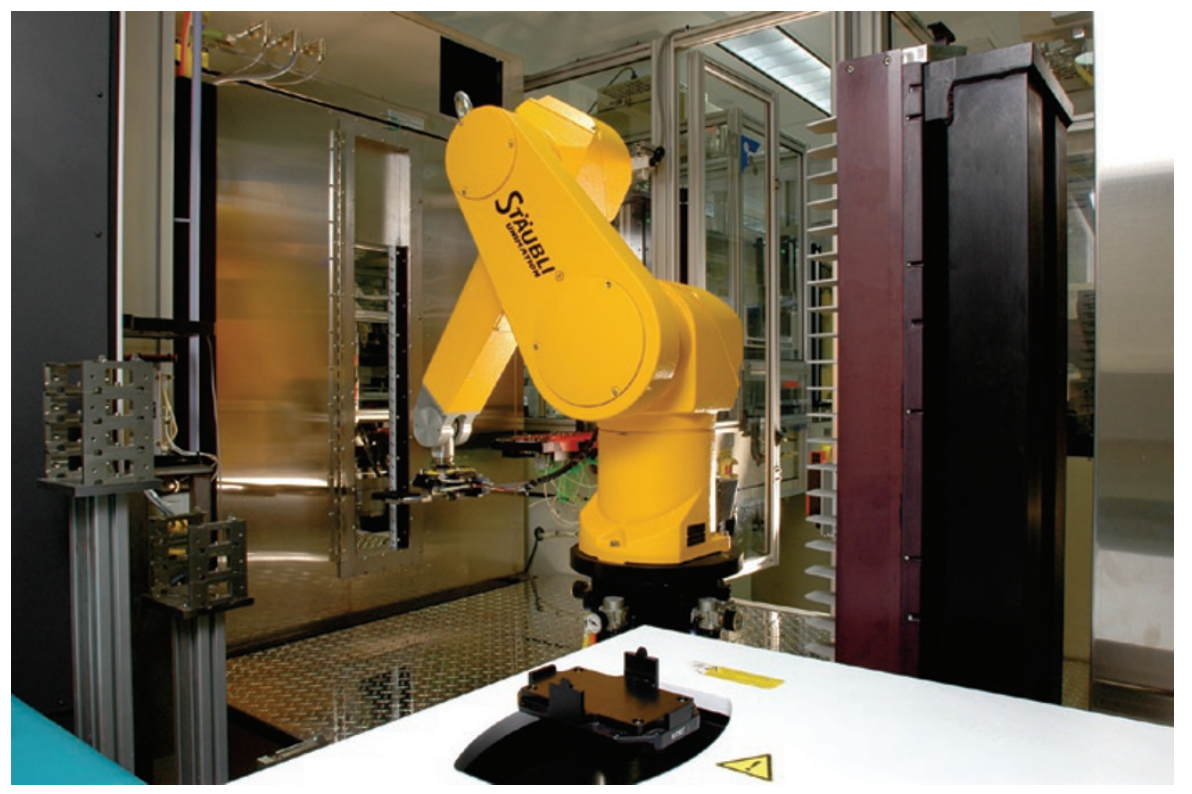

A fully automated system for performing genome-wide RNAi screens. (Courtesy of GNF.) 


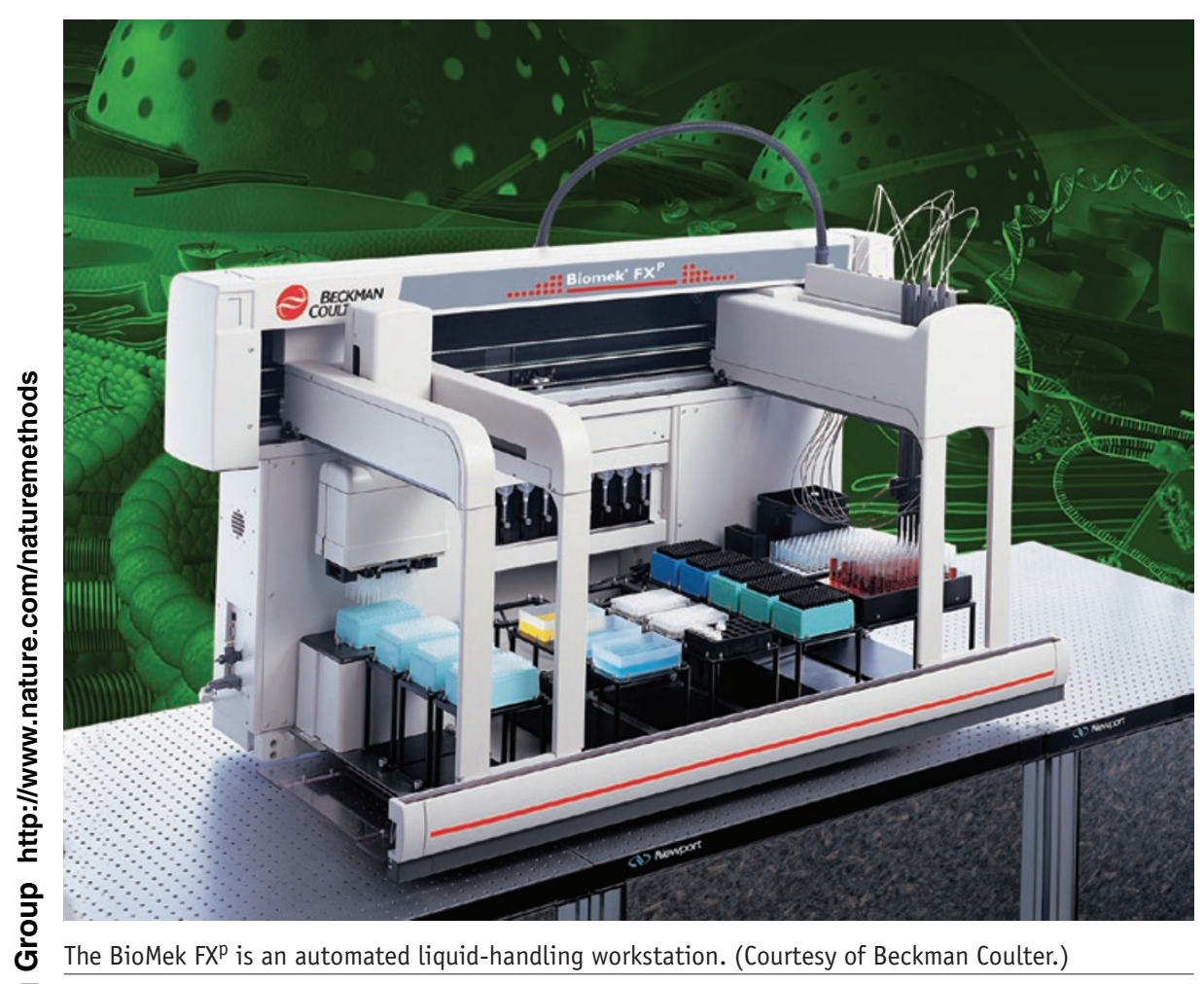

and luminescent assays involving $\mathrm{G}$ protein-coupled receptors and ion channels. Downs notes that the development of the liquid dispenser-plate washer took enormous effort because it has to dispense nanoliter volumes into each well on the plate in a matter of seconds to achieve the necessary throughput. Because each well is so small, the trick, in the end, was to design a new dispenser head to avoid disturbing the cells in the wells or displacing liquids between wells.

Using these robots, scientists at GNF have been performing $50-75$ screens a year-screening 700,000 compounds per day using either biochemical or cell-based assays. And they can also screen subsets of interest from the library at the rate of 100,000 compounds against 100 unique assays in a single experiment. All this means that a screen of two million compounds can be done in less than a week from start to finish-including hit picking, which is performed on a separate robotic system in which an identical compound library has been arrayed into 384-well plates.

Spier notes that they have learned that this level of throughput can be necessary in some cases. "We have done screens of over 2 million compounds and gotten anywhere from zero to tens of promising leads from screen hits, so if you screen any less chemical space, there is lower chance of getting good results to follow up."

\section{My first robot}

"I had always been a one-gene sort of girlworking on one pathway, a few experiments at a time," says Jennifer Rohn, a fellow in the lab group of Buzz Baum at University College London. "Now I am doing highthroughput genome-wide screens, which is a bit hard to get my head around." She uses short interfering RNA (siRNA) screens and automated microscopy to identify genes involved in the shape, polarity and movement of human cells. "For me, I thought the hard part would be the endpoint imaging assay-but it was the setting up that proved most difficult," says Rohn.

To dispense their siRNA library, Rohn and her colleagues relied on Beckman Coulter's Biomek Fx robotic liquid handling system. This system is capable of a variety of liquid-handling applications including nucleic acid preparation, replica plating and chemical screening using either 96- or 384-well plates. For Rohn, using the Biomek Fx presented opportunity and challenge. Seemingly mundane issues like reagent ordering and freezer space took on an entire new meaning. "When you set up one experiment, you go to your fridge and everything is usually there," she says, "but when you are performing a robotic screen, you have to think ahead-instead of 50 microliters of a reagent, you might need 50 liters." In addition, the scale imposed a tedious process of optimization to ensure that the system would dispense the correct amount of liquid per well for all her plates without stopping.

Imaging of cells after addition of siRNA was also automated, using a modified Nikon Instruments Eclipse TE-2000E microscope and Molecular Devices' Metamorph software package for image acquisition and analysis. And by using a Prior Proscan motorized stage in combination with the Metamorph software, which has an integrated autofocus function, an entire plate could be scanned in 11 hours-without operator intervention. Although the image acquisition is automated, Rohn notes that another challenge has been finding a good computer algorithm for analyzing cell morphology. Several other group members are working on this issue, but she still has to spend much time sitting in front of a microscope verifying what the computer 'sees'.

Rohn's introduction to lab automation has sparked her interest in exploring other automated technologies. In the future she plans to collaborate with Jan Ellenberg at the European Molecular Biology Laboratory, who has developed a system for printing siRNAs onto glass, allowing massive confocal time-lapse experiments to be carried out on a genome-wide scale. "I am really looking forward to this because it is a very unique automated system," she says, "although it may prove difficult to import the technology back to our lab." Though implementing these more complex, specialized automated solutions may not be feasible for most labs, many automation experts are willing to collaborate.

"There is the realization now that the technologies that we have created allow far more to be done than we can exploit within the walls of GNF," says Peter Schultz. This has led GNF to create programs to collaborate with academic labs from around the world. And many scientists starting down the road of automated solutions can be encouraged by these offers from the experts-as they present a unique opportunity to apply a level of lab automation to your research that would otherwise be impossible.

Nathan Blow is the Technology Editor

for Nature and Nature Methods.

(n.blow@boston.nature.com). 
TECHNOLOGY FEATURE

\section{SUPPLIERS GUIDE: COMPANIES OFFERING AUTOMATED LABORATORY SYSTEMS}

\section{Company}

Abbott Molecular

Agilent

Applied Biosystems

Aurora Biomed

BD Biosciences

Promega

Protedyne

Qiagen

Rigaku Automation

Roche Applied Science

Tecan Group, Ltd.

Thermo Scientific

Titertek

Torcon Instruments

Velocity 11

Xceed Molecular

Xiril

Zinsser Analytic

\section{Web address}

http://www.abbottmolecular.com

http://www.agilent.com

http://www.appliedbiosystems.com

http://www.aurorabiomed.com

http://www.bdbiosciences.com

http://www.biometra.de

http://www.bio-rad.com

http://www.biotec.co.jp

http://www.biotek.com

http://www.bdal.com

http://www.caliperls.com

http://www.corbettlifescience.com

http://www.cybio-ag.com

http://www.deerac.com

http://www.eppendorf.com

http://www.genetix.com

http://www.genomicsolutions.com

http://www.gilson.com

http://www.gnfsystems.com

http://www.hamiltoncomp.com

http://www.hudsoncontrol.com

http://www.kalypsys-systems.com

http://www.komaxgroup.com

http://www.labcyte.com

http://www.magbio.com

http://www.millipore.com

http://www.moleculardevices.com

http://www.nikoninstruments.com

http://las.perkinelmer.com

http://www.promega.com

http://www.protedyne.com

http://www.qiagen.com

http://www.rigaku.com/automation/

http://www.roche-applied-science.com

http://www.tecan.com

http://www.thermo.com

http://www.titertek.com

http://www.torconinstruments.com

http://www.velocity11.com

http://www.xceedmolecular.com

http://www.xiril.com

http://www.zinsser-analytic.com 